\title{
PERFORMANCE OF ANTIPROTON INJECTION AND EXTRACTION TRANSFER LINES OF THE RECYCLER RING AT FERMILAB
}

\author{
A. Marchionni, T.G. Anderson, G.W. Foster, C. Gattuso, M. Hu, D.E. Johnson, C.J. Johnstone, C. S. \\ Mishra, A.R. Oleck, H. Piekarz, M. Syphers, M.J. Yang, FNAL, Batavia, IL 60510, USA
}

\section{Abstract}

The Recycler Ring, an $8 \mathrm{GeV}$ antiproton accumulator, is being commissioned at Fermilab. Antiproton transfers in and out of the Recycler Ring take place through two transfer lines connecting the Recycler to the Main Injector. Transfer line layout and operation of beam transfers will be described. Particular attention has been paid to injection mismatch effects, in order to limit emittance growth during transfers. A considerable improvement has been achieved by removing vacuum windows, previously present in both transfer lines.

\section{INTRODUCTION}

The Recycler is a fixed $8 \mathrm{GeV}$ kinetic energy antiproton storage ring [1], which makes use of permanent gradient and quadrupole magnets for the ring lattice. It is located in the Main Injector tunnel at a distance of 57" above the Main Injector ring and with the same basic cell geometry. Two transfer lines, MI-22 and MI-32, connect the Recycler to the Main Injector [2]. The MI-22 line is used to transfer antiprotons from the Main Injector into the Recycler, following a clockwise trajectory, and to extract protons from the Recycler into the Main Injector in the counterclockwise direction. Conversely the MI-32 line serves as antiproton extraction line from the Recycler into the Main Injector and as proton injection line into the Recycler for commissioning and tune-ups.

\section{TRANSFER LINE LAYOUT}

The MI-22 and MI-32 lines are positioned on either side of the MI 30 straight section in the Main Injector, where a single kicker at the 304 location is used for both transfer lines. Details on the positioning of the kickers and associated injection and extraction Lambertson magnets in both Main Injector and Recycler rings are summarized in Fig. 1.

At extraction time the circulating beam is kicked horizontally by $1 \mathrm{mrad}$ to enter the magnetic field region of the corresponding Lambertson magnet, which provides a $22 \mathrm{mrad}$ vertical bend of the appropriate sign to initiate the transfer between the two rings. All the Lambertsons follow a same design ( $4 \mathrm{~m}$ long, $1.68 \mathrm{kG}$ ) which makes use of permanent magnet material [3]. Approximately an odd multiple of 90 degree phase advance is mantained between kickers and Lambertsons. The Main Injector Lambertsons are placed in the short (three half-cells) straight sections, while the Lambertson magnets in the Recycler fit easily even in the bend sections.

The distance between each kicker and the associated Lambertson magnet is of the order of $200 \mathrm{~m}$. To reduce the amplitude of the orbit oscillation, induced by the kicker, between the kicker and the Lambertson, an oscillation of approximately half the amplitude and shifted by 180 degrees in phase is created with a time bump. This oscillation is referred to as a counterwave and

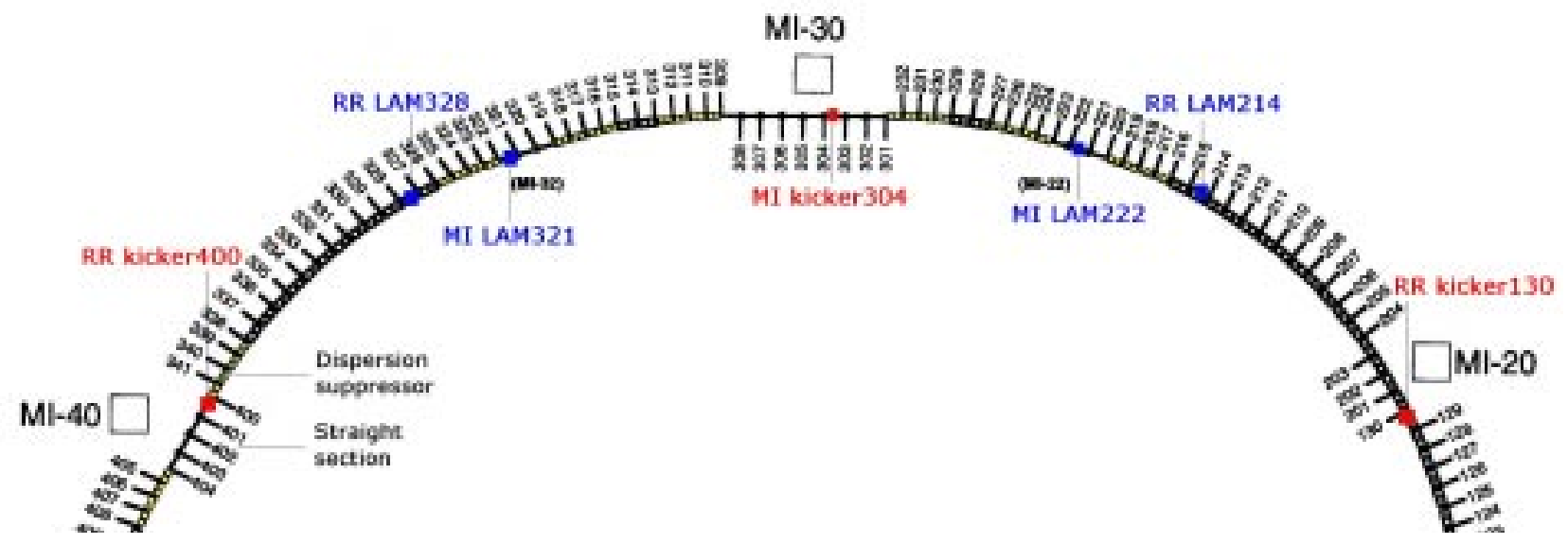

Figure 1: Sketch of locations of kickers and Lambertson magnets in Main Injector (MI) and Recycler (RR) rings. The MI-22 transfer line extends from MI LAM222 to RR LAM214 for approximately 8 half-cells, and the MI-32 line from MI LAM321 to RR LAM328 over about 7 half-cells.

Work supported by the U.S. Department of Energy under contract No. DE-AC02-76CH03000 


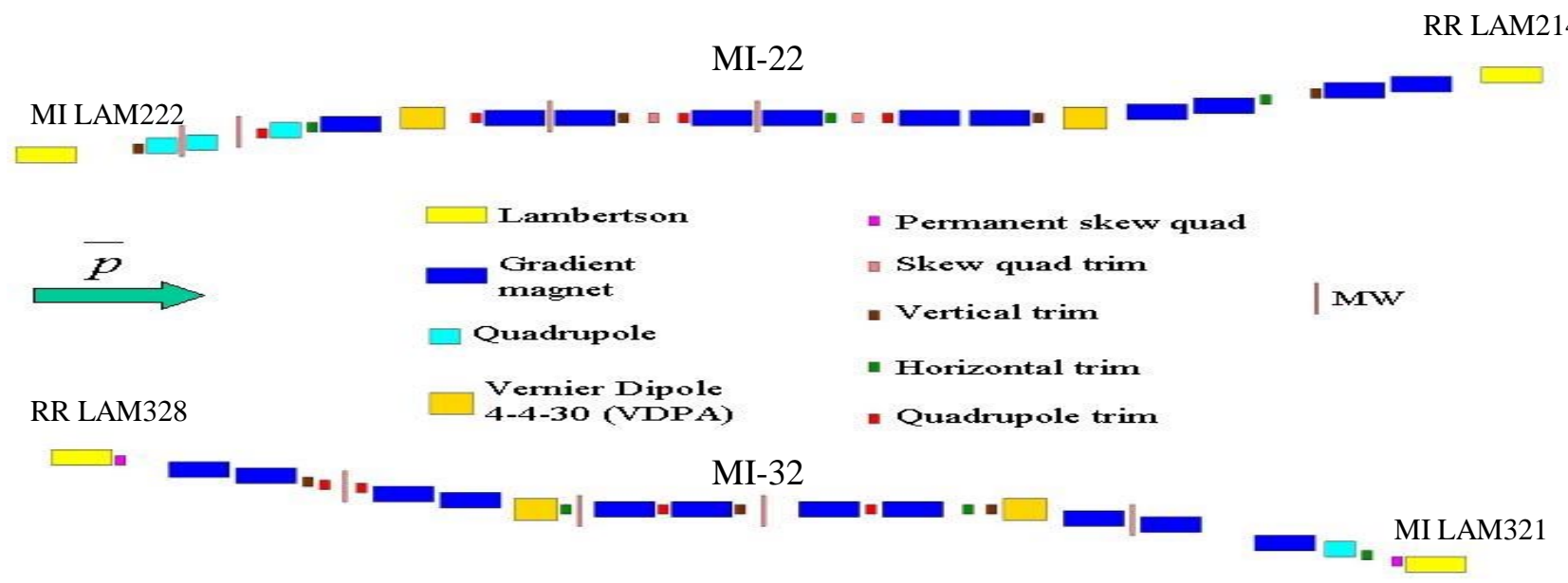

Figure 2: schematic of MI-22 and MI-32 transfer lines

intended to optimize the available horizontal aperture. The resulting orbits of circulating and injected/extracted beams have orbit excursions, opposite in sign, up to a maximum of about $25 \mathrm{~mm}$.

Both MI-22 and MI-32 transfer lines match closely the lattice functions of the Recycler ring and make use of the same quadrupole and gradient permanent magnets of the ring. The use of the gradient magnets is required because the transfer lines for the most part are placed in an arc cell region. The detailed layout is shown in Fig. 2. In addition to the vertical bends provided by the injection and extraction Lambertsons, which are of equal strengths and opposite sign, two additional bends of the same strength and opposite sign are required. They are provided by electromagnets (named VDPA in Fig. 2), which are also used as safety critical devices.

\section{BEAM TRANSFER OPERATION}

Beam is transferred in and out of the Recycler in 2.5 $\mathrm{MHz}$ bunches. All beam transfer operations are driven by clock events. Hardware devices are pre-loaded with all the required settings for manipulations needed to inject or extract antiprotons (or protons for tune-ups). A specific set of clock events are generated to indicate to the hardware which operation to perform. The extraction process begins when the hardware components decode these clock events. Trim dipoles in both rings set the extraction/injection counterwaves just prior to the transfer time.

The transfer line devices generally run in DC mode as they only see single pass beam. Trim dipoles in the transfer lines are used to steer the beam onto the closed orbit of the downstream accelerator to minimize emittance dilution due to steering errors. An automated procedure, which uses a turn by turn beam position detector present in both Recycler and Main Injector rings, extracts position and phase of the injection oscillation and computes new settings of the trim dipoles in order to minimize dilution.
Steering adjustments down to a quarter of a $\mathrm{mm}$ are routinely achieved. Powered quadrupoles were installed in each transfer line for aid in matching lattice functions between transfer lines and both accelerators. These are currently kept off.

Injection and extraction efficiencies larger than $95 \%$ are achievable for beams of about $10 \pi \mathrm{mm}-\mathrm{mrad}$ in $95 \%$ normalized transverse emittance and with $10^{-3}$ fractional momentum spread.

\section{EMITTANCE DILUTION}

During commissioning, beam transfers between Recycler and Main Injector have been showing transverse emittance dilution effects. Emittance dilution measurements were performed with protons injected into the Recycler through the MI-32 line and then transferred back into the Main Injector through the MI-22 line. The emittance of the proton beam was measured in the Main Injector before injection into the Recycler and just after extraction from the Recycler, using the same flying wire system installed in the Main Injector. Measurements showed a cumulative growth, after injection closure, of about $6 \pi \mathrm{mm}-\mathrm{mrad}$, in $95 \%$ normalized transverse emittance, for both planes.

An emittance dilution of this size is unacceptable for antiproton operation. The expected $95 \%$ normalized transverse emittance of cool antiprotons from the Accumulator is about $10 \pi \mathrm{mm}-\mathrm{mrad}$ and that of cold antiprotons from the Recycler should be less than this. The cumulative emittance growth for each transfer, due to all sources, should be kept below $1 \pi$ mm-mrad.

There are four sources of emittance dilution during transfers that need to be considered [4]: betatron function and dispersion mismatch between the transfer line and the ring, steering error into the ring and scattering from vacuum windows, if present. In the following formulae we will always refer to $95 \%$ normalized transverse emittance. 
To get an estimate of the magnitude of the expected dilution due to a betatron function mismatch,

$$
\Delta \varepsilon_{N}=\left[\frac{1}{2}\left(\beta \gamma_{0}+\beta_{0} \gamma-2 \alpha \alpha_{0}\right)-1\right] \cdot \varepsilon_{0},
$$

we compare the design and measured Recycler lattice functions. For an initial 95\% normalized emittance of 10 $\pi$ mm-mrad, we get less than $1 / 2 \pi \mathrm{mm}$-mrad growth.

The dilution due to a dispersion mismatch,

$$
\Delta \varepsilon_{N}=3 \pi(\gamma \beta) \frac{\left(\Delta D_{e q}\right)^{2}}{\beta}\left(\sigma_{p} / p\right)^{2},
$$

does not depend on the initial emittance, but rather on the momentum spread, $\sigma_{\mathrm{p}} / \mathrm{p}$. Assuming a $\sigma_{\mathrm{p}} / \mathrm{p}$ of $10^{-3}$ and a measured dispersion wave of about 0.5 meter, the growth is predicted to be less than $1 / 2 \pi \mathrm{mm}$-mrad.

Currently, we close the injection orbit onto the closed orbit to better than $1 / 4 \mathrm{~mm}$, which produces negligible growth, given by

$$
\Delta \varepsilon_{N}=3 \pi(\gamma \beta)\left(\Delta X_{e q}\right)^{2} / \beta .
$$

Vacuum windows were present in both transfer lines in order to protect the $10^{-10}$ Torr vacuum in the Recycler from the few $10^{-8}$ Torr vacuum in the Main Injector and transfer lines. Due to safety considerations, the Beryllium windows in the original design were replaced by 0.003 " Titanium windows. They were positioned just upstream of the last gradient magnet before injection into the Recycler ring, at a location of maximum vertical betatron function, where $\beta_{\text {Vlatt }}=50 \mathrm{~m}$ and $\beta_{\text {Hlatt }}=15 \mathrm{~m}$.

When an irreducible emittance growth was observed during beam transfers to and from the Recycler, the Titanium windows were reconsidered as a possible cause. Each vacuum window was computed to contribute to a 95\% normalized emittance growth of [5]

$$
\Delta \varepsilon_{N}=3 \pi(\gamma \beta) \beta_{\text {latt }} \vartheta_{\text {rms }}^{2},
$$

where $\theta_{\text {rms }}$ is the projected rms scattering angle due to multiple Coulomb scattering through the material. This gives a growth of $4.2 \pi \mathrm{mm}$-mrad in the vertical plane for each window, which explains most of the measured effect. The observed horizontal/vertical symmetry in emittance growth is likely due to betatron coupling in at least one of the rings or transfer lines.

It was decided to replace the vacuum windows with a differential vacuum pumping system, which was installed in the transfer lines during the January ' 03 shutdown [6]. This system has to deal with a gas load of about $5 \times 10^{-6}$ Torr-1/s from the Main Injector side of the transfer lines and was designed to fit in an available free space extending over a length of about $15 \mathrm{~m}$ in the upper part of the transfer lines, close to the Recycler ring. Pumps are placed with significant lengths of beam pipe between them so that the resulting conductance between pumps is much less than the pumping speed of the pumps. The result is that a relatively small fraction of the gas load at each pump is allowed to flow to the next pump. Three 150 $1 / \mathrm{s}$ ion pumps, spaced about $1.4 \mathrm{~m}$ apart, were installed in a $3 \mathrm{~m}$ long section of the transfer lines closest to the Main Injector. Moving towards the Recycler ring, as the gas load gets reduced, a $150 \mathrm{l} / \mathrm{s}$ titanium sublimation pump (TSP) was placed at about $7.3 \mathrm{~m}$ from the last ion pump. Two more TSPs, $88 \mathrm{l} / \mathrm{s}$ each, were placed $5.6 \mathrm{~m}$ further away, just upstream of the Recycler Lambertson. Next to each TSP small ion pumps of $30 \mathrm{l} / \mathrm{s}$ are mounted. Automatic gate valves protect the Recycler vacuum in the case of vacuum accidents in the Main Injector or transfer lines. The system has been proven to work reliably since installation, providing two orders of magnitude differential pressure, from $10^{-8}$ to $10^{-10}$ Torr.

Emittance growth measurements for the round trip of protons in the Main Injector were taken in this new configuration without vacuum windows. A substantial improvement is observed, as shown in Fig. 3. The residual growth is probably due to betatron function and dispersion mismatch, residual steering errors and kicker waveform defects.

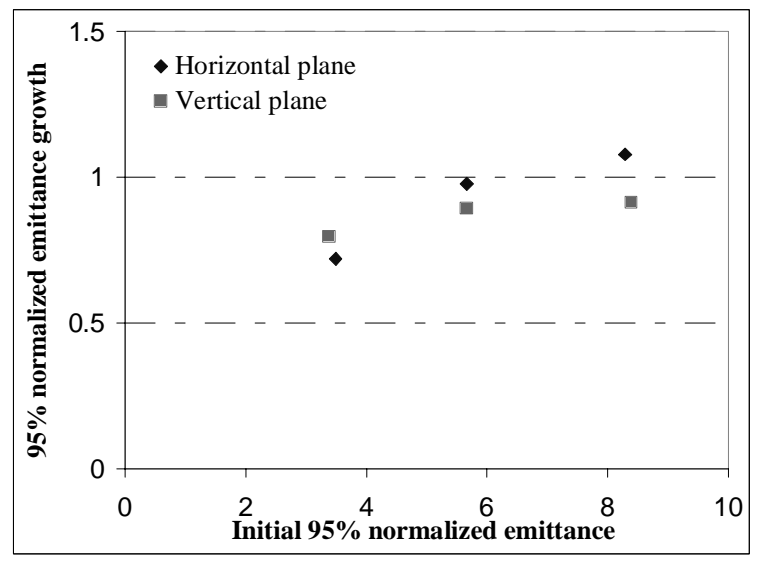

Figure 3: cumulative 95\% normalized transverse emittance growth after vacuum windows were removed

\section{REFERENCES}

[1] G. Jackson, "The Fermilab Recycler Ring Technical Design Report", FERMILAB-TM-1991

[2] D. Johnson, "Revised Recycler pbar transfer lines", Main Injector note 161, December 1997.

[3] M.P. May et al., "The Design and Construction of the Permanent Magnet Lambertson for the Recycler Ring at Fermilab", Proceedings of PAC'97, Vancouver, Canada, 1997.

[4] M.J. Syphers, "Injection Mismatch and Phase Space Dilution", Fermilab note FN-458, June 1987.

[5] M.J. Syphers and C.J. Johnstone, "Vacuum windows in Recycler Transfer Lines", Main Injector note 286, Fermilab Beams-doc-375-v1, September 2002.

[6] T.G. Anderson, A.R. Oleck, H. Piekarz, "Main Injctor-Recycler windowless vacuum system". Report in preparation. 